\title{
Estimation of skin permeation by liquid chromatography
}

\author{
Sara Soriano-Meseguer ${ }^{1}$, Elisabet Fuguet ${ }^{1,2}$, Adriana Port ${ }^{3}$, Martí Rosés ${ }^{1, *}$ \\ ${ }^{1}$ Departament de Química Analítica i Institut de Biomedicina, Universitat de Barcelona, Martí i Franquès 1-11, 08028 \\ Barcelona, Spain \\ 2 Serra Húnter Programme, Generalitat de Catalunya, 08002 Barcelona, Spain \\ ${ }^{3}$ ESTEVE, Parc Científic de Barcelona, Baldiri Reixac 4-8, 08028 Barcelona, Spain \\ *Corresponding Author: E-mail: marti.roses@ub.edu; Tel.: +34-934039275; Fax: +34-934021233
}

Received: February 26, 2018; Revised: April 24, 2018; Available online: May 11, 2018

\begin{abstract}
Dermal absorption is a key process in the drug delivery studies of the pharmaceutical and cosmetic industries, as well as in the fields of dermal toxicology, risk assessment, and the exposure of environmental pollutants. This process is typically described by the skin-water permeability coefficient. However, in vivo determination is laborious and expensive. Thus, in the last few years, the development of prediction models from structure descriptors or subrogation through physico-chemical measurements has gained interest. In the present work, a previous subrogation model based on the chromatographic retention on a common C18 column has been tested for a wide set of drugs with very different chemical nature and having a wide range of permeability values. A total of 65 compounds have been used to establish the correlation between skin permeation and the HPLC retention, corrected by the McGowan volume of the drug. Afterwards it was successfully validated in terms of robustness and prediction ability. Finally, the permeability coefficient was estimated for a set of 29 new drugs, and results compared to the ones obtained by other estimation methods, as well as the available in vitro measured values, with very good agreement.
\end{abstract}

\section{Keywords}

Dermal absorption; High-Performance Liquid Chromatography; Estimation; Skin permeation

\section{Introduction}

The skin is the largest organ of the body, covering about $1.7 \mathrm{~m}^{2}$ and comprising approximately $10 \%$ of the total body mass of an average person. The primary function of the skin is to provide a barrier between the body and the external environment (ultraviolet radiation, chemicals, allergens and microorganisms, and the loss of moisture and body nutrients). Although the skin presents a barrier to the permeation of most compounds, it provides an ideal site for the administration of therapeutic compounds for local and systemic effects [1].

Dermal absorption is a global term that refers to the transport of a chemical from the outer surface of the skin into the skin and the systemic circulation. The outermost layer of the skin, the stratum corneum, controls the dermal absorption process, since substances must initially permeate through it to be absorbed into the body. Hence stratum corneum is considered the rate-determining barrier and the absorption process can only occur by passive diffusion since skin does not have any active transport mechanism $[2,3]$. 
Dermal absorption is a key process in the drug delivery studies of the pharmaceutical and the cosmetic industries as well as in the fields of dermal toxicology, risk assessment and the exposure to environmental pollutants. It is typically described by the skin-water permeability coefficient $\left(k_{\mathrm{p}}\right.$, units in $\left.\mathrm{cm} \mathrm{s}^{-1}\right)$ and can be determined using experimental techniques, both in vivo and in vitro [3]. However, these methods are usually laborious, costly and ethically questionable. For this reason, there is a need for finding methods capable of predicting $k_{\mathrm{p}}$ values in a simple, economic, fast and ethical way.

To predict skin-water permeability coefficients and thus reduce the number of in vivo and in vitro assays, two types of models, empirical and theoretical, can be developed. Empirical models relate the $k_{p}$ values of compounds to physicochemical descriptors, as for example descriptors of hydrophobicity, polarity, solubility, hydrogen bonding, or size [4]. In general, empirical models for skin permeation, as proposed by Patel et al. [5], usually reveal the important roles of lipophilicity and molecular size.

Theoretical models are mechanistic approaches that attempt to simulate the dynamic processes that are involved in skin permeation. They consist of mathematical transport models that relate dermal absorption to parameters like diffusion coefficients and partition coefficients, taking into account factors such as possible routes of penetration and interactions between the permeating chemicals and the skin constituents [6-8].

Linear free-energy relationships (LFERs), a particular type of Quantitative Structure-Activity Relationship (QSAR), are widely used to characterize chemical and biochemical processes. LFERs assume that the free energy change associated with the property of interest is linearly related to solute molecular descriptors. The linear solvation energy relationships (LSERs), proposed by Kamlet et al. [9], are based on the change of free energy due to the solvation process of a solute into a solvent. Among the variety of models based on these principles, the solvation parameter model developed by Abraham [10] is one of the most widely used in order to achieve a better understanding of the type and the relative strength of the chemical interactions that control any solvation process of neutral compounds [11,12]. This model has been successfully applied to characterize many biological processes, including some toxicological and environmental ones, as well as a wide range of physicochemical processes ruled by the passive transport of solutes between two phases. The solvation parameter model $[10,12]$ has been also applied to model the human skin permeation process [12-16]. Abraham and Martins [15] proposed the following equation for characterizing the permeation of neutral solutes from aqueous solution through the human skin,

$\log k_{\mathrm{p}}=-5.246-0.106 E-0.473 \mathrm{~S}-0.473 \mathrm{~A}-3.000 B+2.296 \mathrm{~V}$

$\mathrm{n}=119 \quad \mathrm{R}^{2}=0.832 \quad \mathrm{SD}=0.461 \quad \mathrm{~F}=112$

This equation was obtained through the analysis of 119 compounds of different chemical type, which covered a wide range of $\log k_{\mathrm{p}}$ values. $E, S, A, B$, and $V$ are independent variables and are the solute descriptors proposed by Abraham: $E$ represents the excess molar refraction, $S$ is the solute dipolarity/polarizability, $A$ and $B$ are the solute's effective hydrogen-bond acidity and hydrogen-bond basicity, respectively, and $V$ is the McGowan's solute volume. Eq. 1 shows that size is the only solute property that contributes to an increase in skin permeation (the only positive coefficient).

Over the last few years, the computational models based on calculated descriptors [17-19] have also become very useful and powerful approaches to provide estimations of $k_{\mathrm{p}}$ values. However, skin permeation models based on experimental descriptors are generally preferred when the prediction of skin- 
water permeability of chemicals requires more accuracy and reliability.

In a previous study [20] the ability of different chromatographic systems, basically liquid chromatography (HPLC) and micellar electrokinetic chromatography (MEKC), were assessed to subrogate the permeation of aqueous solutions of neutral solutes through human skin. It was observed that the correlations were improved after introducing the calculated McGowan solute's volume as an additional variable. With the volume correction, the best subrogation was obtained with an HPLC system fitted with a C18 column according to the following equation,

$$
\begin{aligned}
& \log k_{p}=-4.76( \pm 0.18)+1.44( \pm 0.12) \log k-1.16( \pm 0.10) V \\
& \mathrm{n}=27 \quad \mathrm{R}^{2}=0.834 \quad \mathrm{SD}=0.390 \quad \mathrm{~F}=126
\end{aligned}
$$

This system was chosen to establish a methodology for the prediction of human skin permeability of untested compounds by chromatographic measurements. This methodology was developed only for neutral compounds at $\mathrm{pH}$ 7.0. This study has been expanded in the present work to compounds of pharmaceutical interest with a wide range of $\mathrm{p} K_{\mathrm{a}}$ values, containing basic and acidic functions. Additionally, a new methodology based on fast measurements by MS (mass spectrometry) has been developed and established to predict $k_{\mathrm{p}}$ values of drugs of pharmaceutical interest.

\section{Materials and methods}

\section{Instruments}

Chromatographic measurements were performed with an Agilent Technologies 1200 Series instrument equipped with a G1312B binary pump and a G1367D autoinjector. A G1315C DAD was used at $254 \mathrm{~nm}$ for non-ionizable compounds and a UHD 6540 Accurate-Mass Q-TOF detector with electrospray ionization (ESI) source was used for ionizable compounds. A $100 \mathrm{~mm}, 4.6 \mathrm{~mm}$ i.d, $2.6 \mu \mathrm{m}$ octadecylsilica Kinetex EVO C18 analytical column provided by Phenomenex with a core-shell Technology was used for all determinations. This material is stable within the $\mathrm{pH}$ range 1-12.

$\mathrm{pH}$ measurements were done with a MicropH $2001 \mathrm{pH}$-meter (CRISON) with a precision of $\pm 0.01 \mathrm{pH}$ units.

\section{Reagents}

Acetonitrile LCMS grade was purchased from Fluka Analytical VWR (West Chester, PA, USA) and water was purified by a Milli-Q deionizing system from Millipore (Billerica, MA, USA) to a resistivity of $18.2 \mathrm{M} \Omega$. Reagents used to prepare the buffer solutions were sodium phosphate monobasic monohydrate (Sigma-

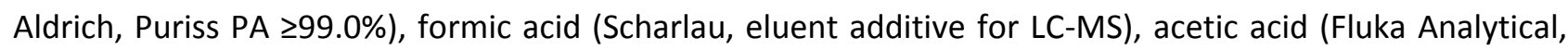
eluent additive for LC-MS), ethylendiamine (Fluka Analytical, Puriss PA $\geq 99.5 \%$ ) and ammonia solution $25 \% \mathrm{w} / \mathrm{w}$ (Sharlau, extrapur, Pharmpur, PH Eur). Most drugs were purchased from Sigma-Aldrich (Steinheim, Germany), Fluka Analytical VWR (West Chester, PA, USA), Riedel-de Haën (Seelze, Germany), Merck (Darmstadt, Germany), Carlo Erba (Milano, Italy) and Baker (Center Valley, PA, USA). Other drugs were synthesized in ESTEVE (Barcelona, Spain) only for the purpose of this study. 


\section{Procedure}

94 solutes of different chemical nature were injected in the HPLC system at 6 different $\mathrm{pH}$ values, between 2 and 11, to obtain the retention factors of the neutral species. The mobile phase composition was $40 \%$ acetonitrile and $60 \%$ aqueous buffer. The buffer solutions were selected according to their compatibility with the detection mode. Volatile buffers were used for mass spectrometry detection. Formic acid, acetic acid and ammonia solution were used at pH 3.0, 5.0 and 9.0, respectively. Ethylendiamine was used at $\mathrm{pH} 7.0$ and 11.0. The buffer concentrations at $\mathrm{pH}$ 3.0, 5.0, 7.0, 9.0 and 11.0 were $10 \mathrm{mM}$ and were adjusted by addition of diluted acetic acid or diluted ammonia. The detection mode was ESI+ or ESI- for ionizable compounds and UV for non-ionizable compounds. For mass spectrometry detection, the compounds were grouped for faster analysis. At pH 2.0, the detection was only performed by UV and a mixture of phosphoric acid and sodium dihydrogenphosphate was used as buffer, at a concentration of $50 \mathrm{mM}$. pH was adjusted with diluted hydrochloric acid. All experiments were done at $25^{\circ} \mathrm{C}$.

Stock solutions of the compounds at $5000 \mathrm{mg} \cdot \mathrm{L}^{-1}$ were prepared by dissolving the appropriate weight or volume in methanol. More diluted solutions were prepared at $100 \mathrm{mg} \cdot \mathrm{L}^{-1}$ by dissolving an aliquot of the previous stock solution in an $\mathrm{ACN}-\mathrm{H}_{2} \mathrm{O}$ mixture (40:60). Isocratic conditions were used at a flow rate of 1 $\mathrm{mL} \mathrm{min}{ }^{-1}$ and the injection volume was $10 \mu \mathrm{L}$. The column hold-up time was measured by injections of an aqueous solution of potassium bromide $\left(0.1 \mathrm{mg} \mathrm{mL}^{-1}\right)$, whose detection was performed by UV at $200 \mathrm{~nm}$. All results were the average of triplicate injections.

The HPLC retention factor ( $k$ ) was calculated according to Eq. 3,

$k=\frac{t_{\mathrm{R}}-t_{0}}{t_{0}-t_{\mathrm{e}}}$

where $t_{\mathrm{R}}$ corresponds to the solute retention time, $t_{0}$ is the column hold-up time determined by an aqueous potassium bromide solution, and $t_{\mathrm{e}}$ is the extra column time determined by an analysis that excludes the chromatographic column, for each different detection system.

\section{Calculation}

Microsoft Excel 2010 was used for all calculations and for multiple linear regression analysis. Solvation parameter model descriptors of the compounds $(E, S, A, B, V)$ were obtained from Percepta [21]. MATLAB $^{\circledR}$ (MathWorks) was used for Principal Components Analysis (PCA).

\section{Model establishment and validation}

\section{Data collection}

The evaluation of the consistency and quality of the experimental skin permeation data used is fundamental to the establishment of a good prediction model. For any QSAR model, experimental data should be produced from standardized experimental procedures, and obtained for a set of chemicals that cover the domain of relevant chemical properties [22].

Several studies that have focused on the determination of skin permeability coefficients [23-25] have pointed out that inter- and intra-variability within experimental $k_{\mathrm{p}}$ values is mainly due to the thickness of the skin and also to the variability inherent to different human donors. In this work, the permeation data employed was selected from the Abraham Database [15], an extensive and carefully examined data set. 65 solutes that belong to different families, with different structural complexity and with varied experimental 
$\log k_{p}$ values were selected to develop this model. Because the permeation coefficients are from water through human skin, it might be expected that acids and bases would be partially ionized in solution depending on the $\mathrm{pH}$ of the solution. Values of $k_{\mathrm{p}}$ for ionized species are smaller than $k_{\mathrm{p}}$ values for the neutral species. In these cases, Abraham adjusted the literature values of the skin-water permeability coefficients for ionization in water and for a temperature of $37^{\circ} \mathrm{C}[15]$.

A different set of 29 solutes was selected from other sources $[5,26,27]$ to predict the $k_{\mathrm{p}}$ values from our model's equation and then compare them to the available literature experimental and/or estimated data.

\section{Model establishment}

A correlation between the experimental $\log k_{p}$ values and the parameters $\log k$ and the McGowan solute's volume ( $V$ ) of the selected compounds was performed through a multiple linear regression analysis. Log $k$ values were the ones measured at a $\mathrm{pH}$ in which the solutes were in the neutral form. The McGowan volume of the compounds can be easily calculated from the empirical formula and the number of single, double, and triple bounds in the molecule. Compounds with a standard residual higher than 2.5 in absolute value were considered outliers.

\section{Model validation}

The model required an internal validation to evaluate its robustness, and an external validation to evaluate its predictive ability. To this aim, the total data set of 65 compounds was divided into two sets, the training set for the internal validation and the test set for the external validation [28]. To construct the two subsets a representation of scores after principal components analysis (PCA) was performed based on the Abraham's molecular descriptors of the compounds. The scores plot distributes the solutes according to their physicochemical properties, so it is easier to do a representative division of the global set of compounds into the two subsets.

The training set, which is aimed at evaluating the robustness of the model, usually has around $50-70 \%$ of the selected compounds. The remaining compounds are used in the test set, which evaluates the predictive ability of the model. The correlation is repeated only with the training set compounds. The results of this second correlation must be compared to the global correlation. If the model is robust, the coefficients of both correlations should be similar. Traditional statistical parameters such as the determination coefficient $\left(R^{2}\right)$, the standard deviation (SD), and the $F$ statistic were also considered to evaluate the robustness of the model. Additionally, the leave-multiple-out cross-validation coefficient, $\mathrm{Q}_{\text {Lмо }}^{2}[29,30]$, was calculated. This coefficient is a parameter that assesses how the results of a statistical analysis will generalize to an independent data set.

The test set was used to perform the external validation of the model, i.e. ensure its predictive ability. The external validation consists in predicting the biological property value of the test set compounds with the training set equation. Then, predicted $\log k_{\mathrm{p}}$ values are plotted against the experimental values. To ensure that the model has a good predictive ability, the slope must be close to 1 and the origin ordinate close to 0 .

\section{Results and Discussion}

Establishment of a model to predict $k_{p}$ values

Table 1 shows the 65 compounds selected to establish the model, their experimental log $k_{\mathrm{p}}$ values, chromatographic retention factors, log $k$ values, and McGowan volumes, $V$. A multiple linear regression 
analysis was done between the experimental $\log k_{\mathrm{p}}$ values and the two parameters $\log k$ and $V$. The set of compounds comprises a wide variety of chemical classes and structural complexity. This is important in order to make the model general enough to predict $k_{\mathrm{p}}$ values of compounds of very different chemical nature. Results are shown in Equation 4 and Figure 1.

Table 1. Experimental $\log k_{\mathrm{p}}$ values, log $k$ values of the neutral species and McGowan solute's volume of the compounds selected to establish the model

\begin{tabular}{|c|c|c|c|}
\hline Compounds & $\log k_{\mathrm{p}}$ & $\log k$ & McGowans's Volume (V) \\
\hline 2,4-Dichlorophenol & -4.30 & 0.556 & 1.0199 \\
\hline 2-Amino-4-nitrophenol & -6.54 & -0.089 & 1.0491 \\
\hline 2-Chlorophenol & -4.56 & 0.237 & 0.8975 \\
\hline 2-Hydroxybenzoic acid (Salycilic acid) & -5.33 & 0.080 & 0.9904 \\
\hline 2-Isopropyl-5-Methylphenol (Thymol) & -4.35 & 0.849 & 1.3387 \\
\hline 2-Naphtol (beta-naphthol) & -4.65 & 0.419 & 1.1441 \\
\hline 2-Nitrophenol & -4.08 & 0.343 & 0.9493 \\
\hline 2-Nitro-p-phenylenediamine & -6.66 & -0.187 & 1.0902 \\
\hline 2-Phenylethanol & -5.20 & 0.003 & 1.0569 \\
\hline 3-Methylphenol (m-Cresol) & -4.89 & 0.172 & 0.9160 \\
\hline 3-Nitrophenol & -5.33 & 0.145 & 0.9493 \\
\hline 4-Amino-2-nitrophenol & -5.91 & -0.010 & 1.0491 \\
\hline 4-Bromophenol & -4.52 & 0.361 & 0.9501 \\
\hline 4-Chloro-3-methylphenol (4-Chloro-m-cresol) & -4.34 & 0.474 & 1.0384 \\
\hline 4-chlorophenol & -4.52 & 0.299 & 0.8975 \\
\hline 4-Ethylphenol & -4.53 & 0.380 & 1.0569 \\
\hline 4-Hydroxybenzyl alcohol & -6.26 & -0.560 & 0.9747 \\
\hline 4-Hydroxy-methylphenylacetate & -5.26 & -0.177 & 1.2722 \\
\hline 4-Hydroxyphenylacetamide & -6.89 & -0.606 & 1.1724 \\
\hline 4-Hydroxy-phenylacetic acid & -6.06 & -0.539 & 1.1313 \\
\hline 4-Methylphenol (p-cresol) & -4.83 & 0.169 & 0.9160 \\
\hline 4-Nitrophenol & -5.33 & 0.091 & 0.9493 \\
\hline 5,5-Diethylbarbituric acid (Barbital) & -7.29 & -0.377 & 1.3739 \\
\hline 5-Ethyl-5-phenylbarbituric acid (Phenobarbital) & -6.68 & -0.059 & 1.6999 \\
\hline 5-Fluorouracil & -6.82 & -0.977 & 0.7693 \\
\hline 8-Methoxypsoralen & -5.12 & 0.376 & 1.4504 \\
\hline Aniline & -4.73 & 0.018 & 0.8162 \\
\hline Aspirin (Acetylsalicylic acid) & -5.69 & -0.165 & 1.2879 \\
\hline Atropine & -7.15 & 0.551 & 2.2820 \\
\hline Benzaldehyde & -3.93 & 0.221 & 0.8730 \\
\hline Benzene & -4.27 & 0.587 & 0.7164 \\
\hline Benzoic acid & -5.15 & -0.073 & 0.9317 \\
\hline Benzyl alcohol & -5.30 & -0.113 & 0.9160 \\
\hline Benzyl nicotinate & -4.87 & 0.569 & 1.6393 \\
\hline Caffeine & -7.08 & -0.487 & 1.3632 \\
\hline Chloropheniramine & -6.14 & 1.009 & 2.2098 \\
\hline
\end{tabular}


Table 1. Continued...

\begin{tabular}{|c|c|c|c|}
\hline Compounds & $\log k_{\mathrm{p}}$ & $\log k$ & McGowans's Volume (V) \\
\hline Codeine & -7.20 & 0.116 & 2.2057 \\
\hline Corticosterone & -6.84 & 0.230 & 2.7389 \\
\hline Dexamethasone & -7.27 & 0.172 & 2.9132 \\
\hline Diclofenac & -5.30 & 0.995 & 2.0250 \\
\hline Diethylcarbamazine & -6.15 & -0.049 & 1.7241 \\
\hline Digitoxin & -8.15 & 0.642 & 5.6938 \\
\hline Ephedrine & -5.49 & 0.314 & 1.4385 \\
\hline Estradiol & -5.61 & 0.534 & 2.1988 \\
\hline Ethylbenzene & -3.00 & 1.065 & 0.9982 \\
\hline Fentanyl & -4.89 & 1.144 & 2.8399 \\
\hline Fluocinonide & -6.33 & 0.904 & 3.4603 \\
\hline Hydrocortisone & -7.22 & -0.055 & 2.7976 \\
\hline Ibuprofen & -5.30 & 1.007 & 1.7771 \\
\hline Indomethacin & -5.39 & 1.001 & 2.5299 \\
\hline Isoquinoline & -5.11 & 0.220 & 1.0443 \\
\hline Lidocaine & -5.42 & 0.764 & 2.0589 \\
\hline Methyl 4-hydroxybenzoate & -5.12 & 0.002 & 1.1313 \\
\hline Methyl phenyl ether (Anisole) & -4.68 & 0.543 & 0.9160 \\
\hline Naproxen & -4.97 & 0.557 & 1.7821 \\
\hline Nicotine & -6.03 & 0.061 & 1.3710 \\
\hline o-Phenylenediamine & -6.70 & -0.313 & 0.9160 \\
\hline Phenol & -5.27 & -0.006 & 0.7751 \\
\hline p-Phenylenediamine & -6.98 & -0.415 & 0.9160 \\
\hline Progesterone & -4.90 & 1.130 & 2.6215 \\
\hline Resorcinol & -6.70 & -0.412 & 0.8338 \\
\hline Scopolamine & -7.90 & -0.036 & 2.2321 \\
\hline Sufentanil & -4.84 & 1.379 & 3.1051 \\
\hline Testosterone & -5.54 & 0.557 & 2.3827 \\
\hline Toluene & -3.64 & 0.826 & 0.8573 \\
\hline
\end{tabular}

$\log k_{\mathrm{p}}=-4.47( \pm 0.12)+1.98( \pm 0.13) \log k-1.02( \pm 0.07) V$

$\mathrm{n}=63 \quad \mathrm{R}=0.826 \quad \mathrm{SD}=0.461 \quad \mathrm{~F}=143$

From the 65 substances, only atropine and chloropheniramine were excluded from the regression analysis because their standard residuals were greater than 2.5 in absolute value. Both coefficients in equation 4 have statistical meaning at a $95 \%$ level of confidence, and log $k$ has the highest contribution to skin permeation. Note that the log $k$ coefficient is almost twice in absolute value the $V$ coefficient. Log $k$ contributes in a positive way, which suggests that a high retention factor implies a better dermal absorption. The $V$ coefficient applies a negative correction to the log $k_{\mathrm{p}}$ value, more important for those compounds with higher volume.

Equation (4) is very similar to the previous equation (2). Direct comparison of the parameters in both cases reveals almost the same values for the $\mathrm{V}$ coefficient and the intercept, and only a slight difference in the log k coefficient. This difference is mainly attributed to the number and variety of compounds studied. Since the present work covers a larger number of compounds and they are more representative of a wide chemical space, it is considered that equation 4 might be more robust than Eq. 2 . 


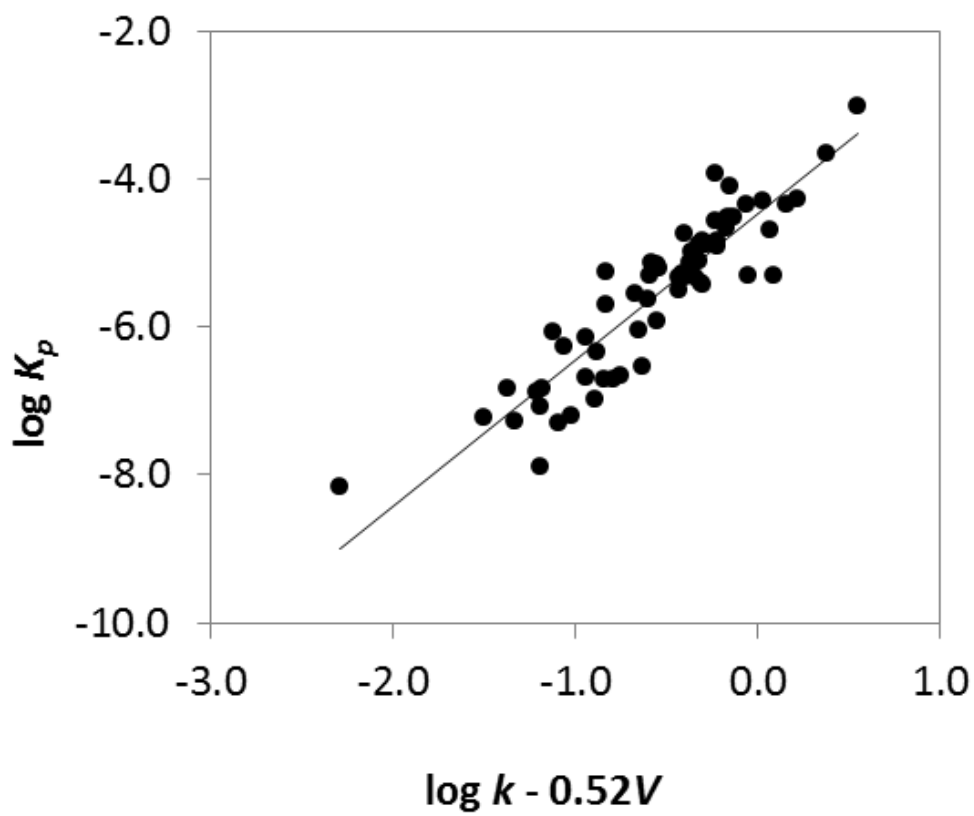

Figure 1. Correlation between $\log k_{\mathrm{p}}$ experimental values and $\log k$ and $V$ of the set of 65 compounds selected to establish the model

As described previously, to validate the model, the global set of 63 compounds was divided into two sets: the training set (42 compounds) and the test set (21 compounds). A multiple linear regression between experimental $\log k_{\mathrm{p}}$ values and $\log k$ and $V$ was performed with the 42 compounds of the training set. Equation 5 and Figure 2a show the results of the regression.

$\log k_{\mathrm{p}}=-4.57( \pm 0.13)+2.06( \pm 0.17) \log k-0.98( \pm 0.08) \mathrm{V}$

(5)

$\mathrm{n}=42$

$\mathrm{R}^{2}=0.832$

SD $=0.450$

$F=97$

$\mathrm{Q}_{\text {LMO }}^{2}=0.832$
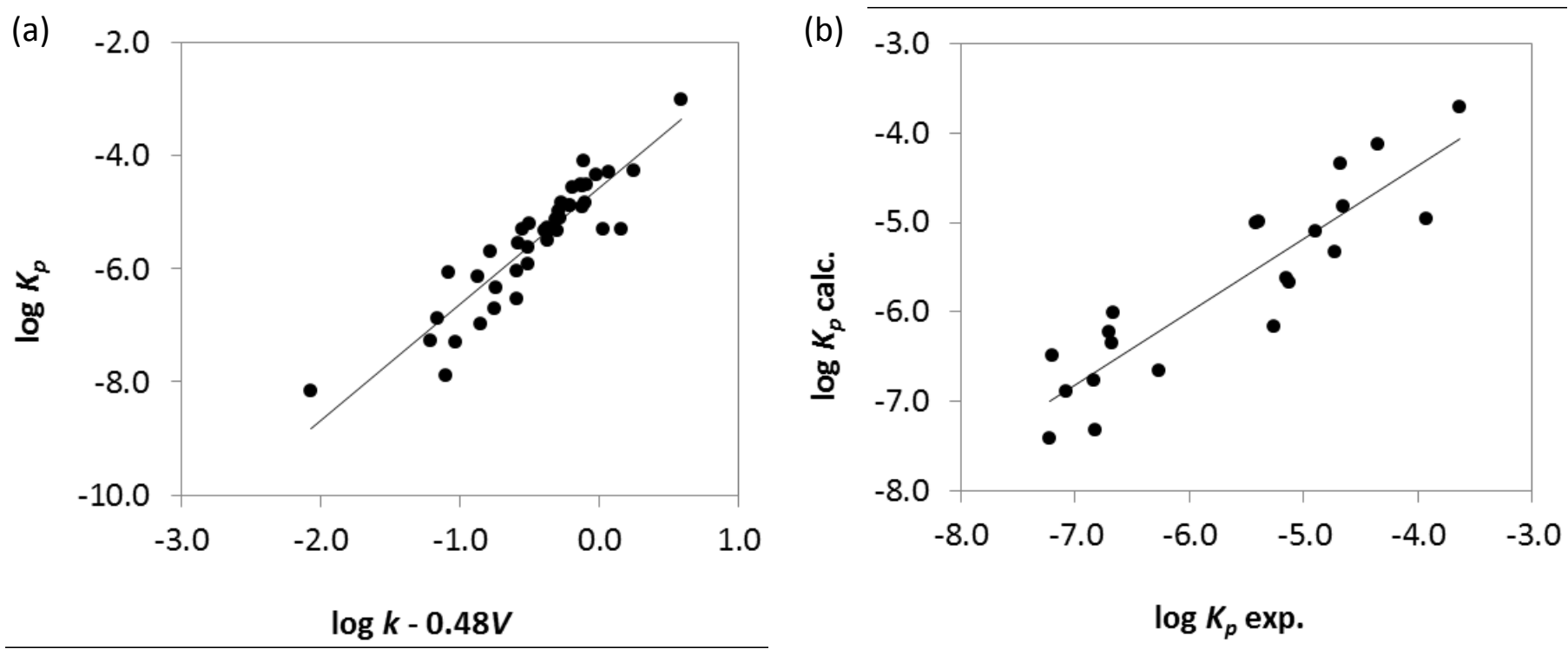

$\log K_{p}$ exp.

Figure 2. (a) Correlation between $\log k_{\mathrm{p}}$ experimental values and $\log k$ and $V$ of the set of compounds selected in the training set. (b) Estimated vs. experimental log $k_{\mathrm{p}}$ values for the compounds of the test set

Comparison of the intercept and the coefficients of Eq. 5 to those of Eq. 4 demonstrate that nearly the same equation is obtained with less compounds, which means that the model is robust, i.e. with fewer points the physical meaning of the coefficients does not change. Adequate determination coefficient, 
standard deviation and $F$ values were obtained. $Q_{\text {LMO }}^{2}$ was higher than 0.6 and it can be ensured that none of the compounds present in the training set has had a big influence in the final model equation.

To perform the external validation, the log $k_{\mathrm{p}}$ values of the 21 compounds of the test set were predicted through the training set equation (Eq. 5). Agreement between experimental and predicted $k_{\mathrm{p}}$ values is shown in Figure $2 \mathrm{~b}$ and through Equation 6:

$\log k_{\text {p, pred }}=-1.08( \pm 0.53)+0.82( \pm 0.09) \log k_{\text {p,exp }}$

$\mathrm{n}=21 \quad \mathrm{R}^{2}=0.807 \quad \mathrm{SD}=0.469 \quad \mathrm{~F}=80 \quad \mathrm{Q}_{\text {LMO }}^{2}=0.983$

According to statistics, the model shows good prediction ability: the slope and intercept of the trend line are not significantly different from unity and zero, respectively, at $95 \%$ confidence level according to Student's $t$ test. The determination coefficient is above 0.6 , the correlation cross-validation coefficient is above 0.5 and Fisher's $F$ parameter is significant.

\section{Prediction of $\log k_{p}$ values}

As a first step, skin permeability coefficients of the neutral species of a set of 20 solutes were determined through the model equation (Eq. 4), after measuring their retention factors in the HPLC system. Table 2 shows the experimental log $k_{\mathrm{p}}$ values $[26,27]$ for these compounds, the values predicted through Eq. 4, and also the values predicted by the solvation parameter model (Eq. 1). For the latter prediction the knowledge of solvation parameter model descriptors of the compounds is necessary. There is quite good agreement between the experimental $\log k_{\mathrm{p}}$ values and the values obtained by Eq. 4 . In most cases the error is around $10 \%$, and is similar to the error presented by the Abraham model (Eq. 1). Figure 3 represents the experimental values against the $\log k_{\mathrm{p}}$ obtained by both models. The solid line represents the theoretical line of slope equal to one and zero intercept. The plot clearly indicates that the accuracy of both models is very similar, with points equally distributed at both sides of the theoretical line, and with similar dispersion. Only famotidine presents an anomalous low log $k_{\mathrm{p}}$ value when predicted by the Abraham model.

In a second step, log $k_{\mathrm{p}}$ values were predicted again through Eq. 4 and then through Eq. 1 for another set of 9 compounds, for which experimental $\log k_{\mathrm{p}}$ values were not known. Only for five of them were the values calculated by Patel et al. (5) available.

As indicated in Table 3, comparison of the data demonstrates that values obtained through chromatographic measurements (Eq. 4) and through the Abraham model (Eq. 1) are in general consistent. However, Patel's predictions are systematically lower compared to the other two models.

Finally log $k_{\mathrm{p}}$ values for four compounds of pharmaceutical interest (capsaicin, oxycodone, tramadol, and warfarin) are proposed for the first time. 
Table 2. Comparison of experimental (in vitro) and predicted $\log k_{\mathrm{p}}$ values

\begin{tabular}{lccccc}
\hline \multirow{2}{*}{ Compounds } & \multicolumn{2}{c}{ In vitro $\log \boldsymbol{k}_{\mathrm{p}}$ values } & & \multicolumn{2}{c}{ Predicted log $\boldsymbol{k}_{\mathrm{p}}$ values } \\
\cline { 2 - 3 } \cline { 5 - 6 } & {$[26]$} & {$[27]$} & & This method & {$[15]$} \\
\hline Aminopyrine & -6.55 & - & -6.45 & -6.97 \\
Antipyrine & -7.74 & - & -6.56 & -7.31 \\
Atrazine & -5.56 & - & & -5.26 & -5.45 \\
Catechol & - & -6.07 & & -5.88 & -5.96 \\
Cortexolone & -7.68 & - & & -6.72 & -5.94 \\
Cortisone & - & -7.38 & & -7.29 & -6.74 \\
Estriol & -7.95 & - & -7.09 & -6.67 \\
Estrone & -6.00 & - & & -5.29 & -5.11 \\
Famotidine & - & -8.35 & & -7.63 & -10.49 \\
Flurbiprofen & -3.40 & - & & -4.60 & -4.09 \\
Griseofulvin & -6.44 & - & & -5.93 & -5.92 \\
Hydroquinone & - & -6.51 & & -6.32 & -6.44 \\
Hydroxyprogesterone & -6.78 & - & -5.80 & -5.08 \\
Ketoprofen & -4.71 & - & & -5.43 & -5.06 \\
Ketorolac & - & -5.80 & & -5.79 & -5.72 \\
Piroxicam & -6.02 & - & & -6.19 & -7.18 \\
Prednisolone & -7.91 & - & -7.46 & -6.90 \\
Pregnenolone & -6.38 & - & -6.05 & -4.93 & -4.70 \\
Propanolol & - & -7.61 & -7.17 & -5.71 \\
Ranitidine & & & & -7.97 \\
\hline
\end{tabular}

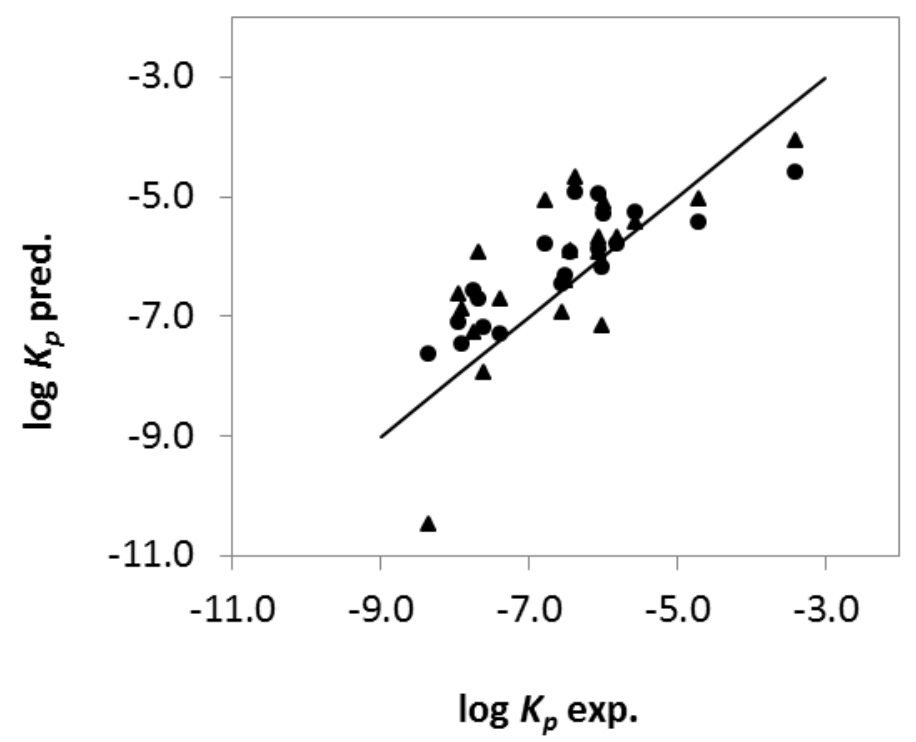

Figure 3. Experimental vs. predicted $\log k_{\mathrm{p}}$ values. For each compound, $\log k_{\mathrm{p}}$ is predicted through the chromatographic model $(\bullet)$ and through the solvation parameter model $(\boldsymbol{\Delta})$ 
Table 3. Prediction of $\log k_{\mathrm{p}}$ values through different estimation models

\begin{tabular}{lccc}
\hline Compounds & This method & [15] & [5] \\
\hline 2-Toluidine & -5.09 & -5.22 & -5.83 \\
3-Xylene & -3.37 & -3.93 & -4.41 \\
Cumene & -3.11 & -3.59 & -6.14 \\
N.N-Dimethylaniline & -4.20 & -4.62 & -5.29 \\
Pyridine & -5.56 & -5.90 & -6.10 \\
Capsaicin & -5.41 & -4.19 & - \\
Oxycodone & -5.89 & -7.05 & - \\
Tramadol & -5.16 & -5.02 & - \\
Warfarin & -5.37 & -5.25 & - \\
\hline
\end{tabular}

\section{Conclusions}

A methodology has been developed for the prediction of skin permeability of neutral species. The method is based on measurements on an HPLC system fitted with a C18 column, coupled to UV-vis and MS detection. The model has been established with a set of 63 compounds with different chemical natures and structural complexity, which ensures the applicability of the model to a wide range of compounds. The chromatographic retention factor is the parameter that makes the highest contribution to the calculation of the permeation through skin, the $k_{p}$ value being greater as the retention increases. On the contrary, the McGowan volume decreases the permeation value. The model has been validated, showing both good robustness and good prediction ability. The prediction ability has been further tested by comparison of the results to other prediction models (Abraham and Patel). Results from this work are similar (both in accuracy and precision) to the results obtained through Abraham's model. However, the permeation coefficients obtained through Patel's model seem to be slightly lower. The main advantage of the present methodology is that $k_{\mathrm{p}}$ can be directly obtained from simple chromatographic retention measurements and the McGowan's molecular volume of the compounds, without the need of additional molecular descriptors calculation and/or determination.

Acknowledgements: We thank Magda Bordas for her expert contribution to the analytical studies and with Edmundo Ortega for their excellent technical assistance. We also thank Carmen Almansa for her very helpful discussion and comments. Financial support from the Generalitat de Catalunya (Project DI-2014 modality DI-ESP, resolution ECO/1426/2014) and the Ministerio de Economia y Competitividad from the Spanish Government (CTQ2014-56253-P) is acknowledged.

\section{References}

[1] H.A.E. Benson, A.C. Watkinson. Topical and Transdermal Drug Delivery: Principles and Practice. Wiley (2012).

[2] K. Hoang. Dermal Exposure Assessment: Principles and Applications. Washington: Environmental Protection Agency, Office of health and Environmental Assessment (1992).

[3] J. Kielhorn, S. Melching-Kollmuss, I. Mangelsdorf. Environmental Health Criteria 235: Dermal Absorption. Geneva: World Healt Organization Press (2006).

[4] S. Geinoz, R.H. Guy, B. Testa, P. Carrupt. Quantitative structure-permeation relationships (QSPeRs) to predict skin permeation: a critical evaluation. Pharmaceutical Research 21 (2004) 83-92. 
[5] H. Patel, W. ten Berge, M.T.D. Cronin. Quantitative structure-activity relationships (QSARs) for the prediction of skin permeation of exogenous chemicals. Chemosphere 48(6) (2002) 603-613.

[6] S. Mitragotri, Y.G. Anissimov, A.L. Bunge, H.F. Frasch, R.H. Guy, J. Hadgraft, G.B. Kasting, M.E. Lane, M.S. Roberts. Mathematical models of skin permeability: an overview. International Journal of Pharmaceutics 418 (2011) 115-129.

[7] A. Tezel, S. Mitragotri. On the origin of size-dependent tortuosity for permeation of hydrophilic solutes across the stratum corneum. Journal of Controlled Release 86 (2003) 183-186.

[8] A. Wilschut, W. ten Berge, P.J. Robinson, T.E. McKone. Estimating skin permeation. The validation of five mathematical skin permeation models. Chemosphere 30 (1995) 1275-1296.

[9] M.J. Kamlet, R.M. Doherty, J.L.M. Abboud, M.H. Abraham, R.W. Taft. Solubility: a new look. Chemtech 16(9) (1986) 566-576

[10] M.H. Abraham. Scales of solute hydrogen-bonding: their construction and application to physicochemical and biochemical processes. Chemical Society Reviews 22 (1993) 73-83.

[11] M. Vitha, P.W. Carr. The chemical interpretation and practice of linear solvation energy relationships in chromatography. Journal of Chromatography A 1126 (2006) 143-194.

[12] M.H. Abraham, H.S. Chadha, F. Martins, R.C. Mitchell, M.W. Bradbury, J.A. Gratton. Hydrogen bonding part 46: a review of the correlation and prediction of transport properties by an LFER method: physicochemical properties, brain penetration and skin permeability. Pest Management Science 55 (1999) 78-88.

[13] M.H. Abraham, Chadha, H.S., Mitchell, R.C. (1995b). The factors that influence skin penetration of solutes. Journal of Pharmacy and Pharmacology, 47, 8-16.

[14] M.H. Abraham, F. Martins, R.C. Mitchell. Algorithms for skin permeability using hydrogen bond descriptors: the problem of steroids. Journal of Pharmacy and Pharmacology 49 (1997) 858-865.

[15] M.H. Abraham, F. Martins. Human skin permeation and partition: general linear free-energy relationship analyses. Journal of Pharmaceutical Sciences 93 (2004) 1508-1523.

[16] K. Zhang, M. Chen, G.K.E. Scriba, M.H. Abraham, A. Fahr, X. Liu. Human skin permeation of neutral species and ionic species: Extended linear free-energy relationship analyses. Journal of Pharmaceutical Sciences 101 (2012) 2034-2044.

[17] B.J. Neely, S.V. Madihally, R.L. Robinson Jr, K.A.M. Gasem. Nonlinear quantitative structureproperty relationship modeling of skin permeation coefficient. Journal of Pharmaceutical Sciences, 98 (2009) 4069-4084.

[18] P. Buchwald, N. Bodor. A simple, predictive, structure-based skin permeability model. Journal of Pharmacy and Pharmacology 53 (2001) 1087-1098.

[19] D. Neumann, O. Kohlbacher, C. Merkwirth, T. Lengauer. A fully computational model for predicting percutaneous drug absorption. Journal of Chemical Information and Modeling 46 (2006) 424-429.

[20] M. Hidalgo-Rodríguez, S. Soriano-Meseguer, E. Fuguet, C. Ràfols, M. Rosés. Evaluation of the suitability of chromatographic systems to predict human skin permeation of neutral compounds. European Journal of Pharmaceutical Sciences 50(5) (2013) 557-568.

[21] ACD/Percepta, www.acdlabs.com, Advanced Chemistry Development, Inc., Release (Build 2726, 5 May 2014).

[22] A.D. Jones, I.P. Dick, J.W. Cherrie, M.T.D. Cronin, J.J.M. van de Sandt, D.J. Esdaile, S. Iyengar, W. ten Berge, S.C. Wilkinson, C.S. Roper, S. Semple, C. de Heer, F.M. Williams. CEFIC workshop on methods to determine dermal permeation for human risk assessment. European Chemical Industry Council, December (2004) pp 1-86

[23] J.J. van de Sandt, J.A. van Burgsteden, S. Cage, P.L. Carmichael, I. Dick, S. Kenyon, G. Korinth, F. Larese, J.C. Limasset, W.J. Maas. In vitro predictions of skin absorption of caffeine, testosterone, and benzoic acid: A multi-centre comparison study. Regulatory Toxicology and Pharmacology 39 (2004) 271-281. 
[24] S.C. Wilkinson, W.J.M. Maas, J.B. Nielsen, L.C. Greaves, J.J.M. van de Sandt, F.M. Williams. Interactions of skin thickness and physicochemical properties of test compounds in percutaneous penetration studies. International Archives of Occupational and Environmental Health 79 (2006) 405-413.

[25] F. Heuber, F. Ouvrard-Baraton, J.P. Biesse, P. Courtellemont, C.M. Vincent, J.P. Marty. Pig ear skin as a model for in vitro percutaneous absorption studies: Preliminary results of an inter-laboratory validation. In: K.R. Brain, K.A. Walters, editors. Perspectives in percutaneous penetration, Vol. 6 a. Cardiff: STS Publishing Ltd (1998).

[26] B.E. Vecchia, A.L. Bunge. Transdermal drug delivery (pp. 25-55). New York: Marcel Dekker (2003).

[27] H. Baba, J. Takahara, H. Mamitsuka. In silico predictions of human skin permeability using nonlinear quantitative structure-property relationships models. Pharmaceutical Research 32 (2015) 23602371.

[28] K. Roy. On some aspects of validation of predictive quantitative structure-activity relationship models. Expert Opinion on Drug Discovery 2(12) (2007) 1567-1577.

[29] A. Tropsha, P. Gramatica, V. Gombar. The Importance of Being Earnest: Validation is the Absolute Essential for Successful Application and Interpretation of QSPR Models. QSAR \& Combinatorial Science 22(1) (2003) 69-77. 